Gynäkologische Endokrinologie 2011 . 9:143-143

DOI 10.1007/s10304-011-0412-3

Online publiziert: 10. September 2011

(c) Springer-Verlag 2011

\author{
O. Ortmann ${ }^{1} \cdot$ L. Kiesel ${ }^{2}$ \\ ${ }^{1}$ Klinik für Frauenheilkunde und Geburtshilfe, Caritas-Krankenhaus St. Josef, \\ Universität Regensburg \\ ${ }^{2}$ Klinik und Poliklinik für Frauenheilkunde und Geburtshilfe, \\ Universitätsklinikum Münster
}

\title{
Menopause und Frauengesundheit
}

häufig eine langfristige Gabe von Östrogenen empfohlen, ggf. in Kombination mit Gestagenen in der Postmenopause. In diesem Zusammenhang ist allerdings zu bedenken, dass insbesondere ein langfristiger Einsatz von Sexualsteroiden auch Risiken bergen kann. Nutzen und Risiken werden bis heute kontrovers diskutiert. Mit der S3Leitlinie zur Hormontherapie in der Periund Postmenopause wurde ein praxistaugliches Instrument entwickelt, das eine Nutzen-Risiko-Bewertung ermöglicht. Im Beitrag von C. Lattrich u. O. Ortmann wird diese Leitlinie in kurzer Form dargestellt.

Da eine Hormontherapie in der Periund Postmenopause auf diverse klinische Endpunkte wirken kann, ist eine fundierte Kenntnis der Zusammenhänge von besonderer Bedeutung. Auch wenn heute präventive Therapieansätze etwas in den Hintergrund gerückt sind, ist die Hormontherapie nach wie vor eine der wichtigsten und wirksamsten Therapieoptionen zur Behandlung bestimmter Symptome und Erkrankungen in der Peri- und Postmenopause. Nur eine individualisierte Therapie, die das Gesundheitsprofil der betroffenen Frauen, ihre Bedürfnisse und die Therapieziele klar definiert, ermöglicht eine adäquate Behandlung. In der Beratung der Patientinnen ist ein Gespräch über Nutzen und Risiken der Therapie, das an die individuelle Situation angepasst werden sollte, von besonderer Bedeutung. Bestimmte Risiken werden zum Teil bagatellisiert, andere zum Teil überbewertet. Es ist daher wichtig, Frauen ein realistisches Bild der Zusammenhänge zu vermitteln. Große Bedenken bestehen häufig im Zusammenhang mit der Entstehung von gynäkologischen Krebserkrankungen. M. Wolf $\mathrm{u}$.
L. Kiesel stellen diese Risiken in ihrem Beitrag in adäquater Weise dar.

Fortschritte sind nicht nur im Umgang mit den verschiedenen Optionen der Hormontherapie erforderlich. Auch die Einführung neuer Therapieregime kann sinnvoll sein und unser therapeutisches Armamentarium erweitern. I. Wiegratz beschreibt in ihrem Beitrag die Rolle einer ultraniedrig dosierten Hormonersatztherapie in der Peri- und Postmenopause. Zielführend ist ein differenzierter Umgang mit dieser Therapieoption unter besonderer Berücksichtigung der Erwartungen von Rat suchenden Frauen.

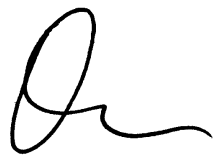

Prof. Dr. Olaf Ortmann

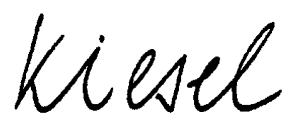

Prof. Dr. Ludwig Kiesel

\section{Korrespondenzadresse \\ Prof. Dr. 0. Ortmann}

Klinik für Frauenheilkunde und Geburtshilfe, Caritas-Krankenhaus St. Josef,

Universität Regensburg

Landshuter Str. 65, 93053 Regensburg

olaf.ortmann@klinik.uni-regensburg.de

Prof. Dr. L. Kiesel

Klinik und Poliklinik für Frauenheilkunde und Geburtshilfe,

Universitätsklinikum Münster

Albert-Schweitzer-Str. 33, 48149 Münster

L.Kiesel@uni-muenster.de therapie in der Vergangenheit auch ein pra ventives Ziel verfolgt. Infolgedessen wurde 